\title{
Morphological Difference between Solution-Cast and Melt-Quenched Crystalline-Amorphous Diblock Copolymers
}

\author{
Awaludin RoHAdi, Satoshi TANImoto, Shintaro SASAKI, \\ and Shuichi NOJIMA ${ }^{\dagger}$ \\ School of Materials Science, Japan Advanced Institute of Science \\ and Technology (JAIST), Tatsunokuchi, Ishikawa 923-1292, Japan
}

(Received March 27, 2000; Accepted June 19, 2000)

\begin{abstract}
The morphology formed in poly $(\varepsilon$-caprolactone)-block-polybutadiene (PCL- $b$-PB) copolymers by casting the toluene solution was investigated by small-angle X-Ray scattering (SAXS) and differential scanning calorimetry (DSC) as a function of total molecular weight $M_{n}$ and crystallization temperature $T_{\mathrm{c}}$, and compared with the morphology formed by quenching from the microphase-separated melt. The lamellar morphology was observed at any $T_{\mathrm{c}}$ in the solution-cast PCL- $b$-PB with lower $M_{n}\left(M_{n} \leq 30000\right)$, and the repeating distance of this morphology was little affected by $T_{\mathrm{c}}$ while it increased significantly with increasing $T_{\mathrm{c}}$ for the quenched PCL- $b$-PB. The solution-cast PCL- $b$-PB with higher $M_{n}\left(M_{n} \geq 44000\right)$ had the lamellar morphology or crystallized microdomain structure (i.e., a microdomain structure in which the PCL block partially crystallized) depending on $T_{\mathrm{c}}$ though the crystallized microdomain structure was always observed in the quenched samples. The PCL crystallinity $\chi_{\mathrm{c}}(i . e$., the weight fraction of crystallized PCL blocks against total PCL blocks in the system) was $0.63-0.79$ in the lamellar morphology while it was considerably low in the crystallized microdomain structure $\left(\chi_{\mathrm{c}}<0.23\right)$, suggesting the substantial difficulty of crystallization within the curved microdomain. The mechanism of morphology formation in the solution-cast samples is discussed by considering the competition between microphase separation and crystallization during the solvent evaporation at each $T_{\mathrm{c}}$ and $M_{n}$. KEY WORDS Crystalline-Amorphous Diblock Copolymer/Solution-Casting Method/Crystallization / Microphase Separation / Morphology Formation /
\end{abstract}

Crystalline-amorphous diblock copolymers form a lamellar morphology, an alternating structure consisting of lamellar crystals and amorphous layers, when they crystallize by quenching from the weakly segregated microdomain structure. ${ }^{1}$ The resulting lamellar morphology is believed to be close to the equilibrium morphology predicted by recent theories ${ }^{2,3}$ when the molecular weight of constituent copolymers is not high and simultaneously the glass transition temperature of the amorphous block is enough low. However, it is sometimes observed in such block copolymers that the repeating distance of the lamellar morphology $L$ is significantly dependent on crystallization temperature $T_{\mathrm{c}}{ }^{4-7} ; L$ increases steadily with increasing $T_{\mathrm{c}}$, as usually observed in the crystallization of homopolymers. This means that the lamellar morphology formed by quenching is in nonequilibrium though it may be close to the equilibrium morphology.

The morphology formed by the solution-casting method has been investigated for crystalline-amorphous block copolymers, ${ }^{8-17}$ and the morphological control is also tested for some block copolymers, where the solvent nature determines the final morphology through an interplay between crystallization and microphase separation. When we use the solvent which is good only for the crystalline block, for example, the microdomain structure is formed first during the solvent evaporation and eventually the crystallization takes place partially within this structure. ${ }^{8}$ Thus, the solution-casting method has a possibility to introduce a wide variety of path-dependent morphologies in crystalline-amorphous block copolymer systems.

If we choose the solvent which is good (or non- selective) for both blocks it will be possible to get the equilibrium lamellar morphology by the slow evaporation of the solvent, which is not attained by quenching from the microphase separated melt. There are, however, few studies to investigate the lamellar morphology formed by the solvent evaporation as a function of total molecular weight $M_{n}$ and $T_{\mathrm{c}}$. In the present study, we crystallize poly( $\mathcal{E}$-caprolactone)-block-polybutadiene (PCL- $b$-PB) copolymers by casting the toluene solution at $T_{\mathrm{c}}$ and the final morphology is investigated by smallangle X-Ray scattering (SAXS) and differential scanning calorimetry (DSC). By comparing the morphology with that formed by quenching from the microphaseseparated melt, ${ }^{18}$ we elucidate the morphological difference between two methods. We expect that the high molecular weight PCL- $b$-PB, which has a strongly segregated microdomain structure in the melt and therefore the PCL block crystallizes partially within it by quenching, takes an equilibrium lamellar morphology by the solution-casting method.

\section{EXPERIMENTAL}

\section{Materials}

Poly( $\varepsilon$-caprolactone)-block-polybutadiene (PCL- $b$-PB) copolymers were synthesized by a successive anionic polymerization under vacuum with $n$-buthyllithium as an initiator. Details of the synthesis, purification, and molecular characterization are described elsewhere. ${ }^{19,20}$ The molecular characteristics of PCL- $b$-PB copolymers are summarized in Table I. The small PCL fraction ranging from 0.10 to 0.19 makes us expect the spherical or cylindrical microdomain structure with the PCL block

\footnotetext{
${ }^{\dagger}$ To whom correspondence should be addressed (Phone: +81-761-51-1601, Fax: +81-761-51-1149, E-mail: nojima@jaist.ac.jp).
} 
Table I. Characterization of PCL- $b$-PB copolymers used in this study

\begin{tabular}{|c|c|c|c|c|c|c|c|}
\hline \multirow{2}{*}{ Notation } & \multirow{2}{*}{ Total $M_{n}^{\mathrm{a}}$} & \multirow{2}{*}{$M_{w} / M_{n}^{b}$} & \multirow{2}{*}{$\frac{\mathrm{PCL}: \mathrm{PB}^{\mathrm{c}}}{\text { vol } \%}$} & \multicolumn{3}{|c|}{ Microstructure of PB Chain $\mathrm{c} / \mathrm{mol} \%$} & \multirow{2}{*}{$T_{\mathrm{m}}{ }^{\mathrm{d}}\left(T_{\mathrm{c}}\right) / /^{\circ} \mathrm{C}$} \\
\hline & & & & cis- 1,4 & trans $-1,4$ & 1,2-linkage & \\
\hline M11 & 11000 & 1.15 & $19: 81$ & 34 & 49 & 17 & $51(30)$ \\
\hline M30 & 30000 & 1.11 & $11: 89$ & 35 & 59 & 6 & $54(10)$ \\
\hline M44 & 44000 & 1.07 & $10: 90$ & 34 & 60 & 6 & $54(10)$ \\
\hline M62 & 62000 & 1.13 & $13: 87$ & 38 & 55 & 7 & $57(10)$ \\
\hline M140 & 140000 & 1.43 & $15: 85$ & 36 & 58 & 6 & $59(10)$ \\
\hline
\end{tabular}

${ }^{\mathrm{a}}$ Determined by VPO or Membrane Osmometry. ${ }^{\mathrm{b}}$ Determined by GPC. ${ }^{\mathrm{c}}$ Determined by ${ }^{1} \mathrm{H}$ NMR. ${ }^{\mathrm{d}}$ Determined by DSC.

inside for all the copolymers.

Toluene, good for both the blocks, was used for the casting solvent, and ca. $5 \mathrm{wt} \%$ solution was cast at each $T_{\mathrm{c}}$ ranging from -20 to $45^{\circ} \mathrm{C}$. The solvent was evaporated under the atmosphere and finally under the reduced pressure.

\section{SAXS Measurements}

The SAXS measurements were performed with a point focusing optics and a one-dimensional position sensitive proportional counter with an effective length of $10 \mathrm{~cm}$. Details of the optics, instrumentation, and data processing are described elsewhere. ${ }^{21-23}$ The SAXS curve after various corrections was finally obtained as a function of wave number $s$ defined as $s=(2 / \lambda) \sin \theta$, where $\lambda$ is the wave length of the incident X-Ray $(=0.1542 \mathrm{~nm})$ and $2 \theta$ is the scattering angle. We evaluated the repeating distance of the resulting morphology $L\left(=1 / s^{*}, s^{*}\right.$ is the angular position of the intensity peak) and full width at half maximum (FWHM) of the intensity peak, where the SAXS intensity from the lamellar morphology was further corrected by multiplying the Lorentz factor $\left(=4 \pi s^{2}\right)$. The values of $L$ and FWHM thus evaluated were compared with those for the quenched samples.

\section{DSC Measurements}

A Perkin-Elmer DSC 7 or Pyris 1 was used to evaluate the melting temperature $T_{\mathrm{m}}$ and PCL crystallinity $\chi_{\mathrm{c}}$ (i.e., the weight fraction of crystallized PCL blocks against total PCL blocks in the system) of the sample. The DSC curve was obtained at a heating rate of $5^{\circ} \mathrm{C}$ $\min ^{-1}$ for all the samples investigated. $T_{\mathrm{m}}$ was defined as the maximum temperature of the endothermic peak and $\chi_{c}$ was calculated by $\chi_{\mathrm{c}}=\Delta H /\left(\Delta H^{*} \phi_{\mathrm{PCL}}^{\prime}\right)$, where $\Delta H$ and $\Delta H^{*}$ are the heat of fusion of the sample and perfect PCL crystal $\left(=135.44 \mathrm{~J} \mathrm{~g}^{-1}\right)^{24}$ and $\phi_{\text {PCL }}^{\prime}$ is the weight fraction of PCL blocks in the sample.

\section{RESULTS}

\section{SAXS Measurements}

Figure 1 shows typical SAXS curves of M11, M30, and M62 crystallized by the solution-cast (denoted by S) and quenched $(\mathrm{Q})$ methods. The SAXS curves are similar for M11 (and also M30) prepared by both methods though the angular position of the intensity peak is slightly different. Both the SAXS curves have a faint second peak or shoulder, the position of which exactly corresponds to double of the first peak position, suggesting that the lamellar morphology is formed in M11 and M30 by both the methods though the detailed structure may be different. The formation of the lamellar morphology in the

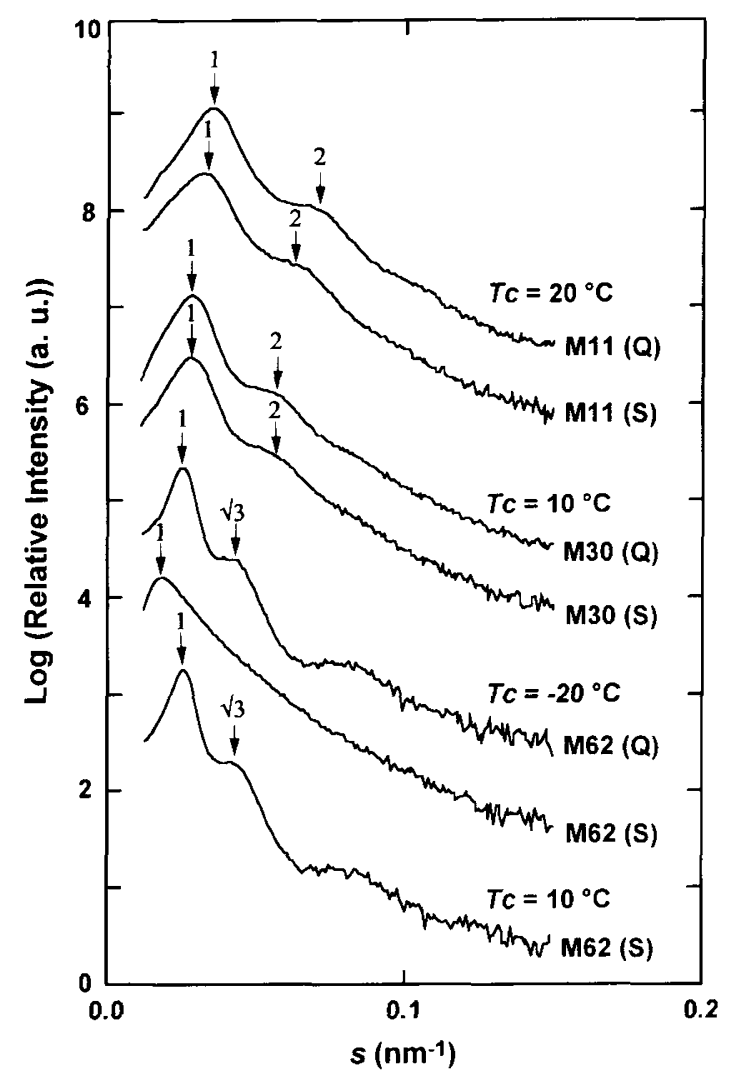

Figure 1. SAXS curves of M11, M30, and M62 cast from the toluene solution (denoted by $S$ ) and quenched from the microphase separated melt $(Q)$ plotted logarithmically against $s(=(2 / \lambda) \sin$ $\theta, 2 \theta$ is the scattering angle and $\lambda$ is the $\mathrm{X}$-Ray wave length $(=0.1542 \mathrm{~nm})) . T_{\mathrm{c}}$ represents the crystallization temperature.

quenched M11 and M30 is ascribed to the disruption of weakly segregated microdomain structures by the strong driving force of crystallization, ${ }^{4,5}$ and therefore the lamellar morphology formed is not always in equilibrium.

The SAXS curve of the solution-cast M62 is extremely different from that of the quenched M62 at $T_{\mathrm{c}}=-20^{\circ} \mathrm{C}$; the quenched $\mathrm{M} 62$ retains the microdomain structure after the crystallization of PCL blocks, so that the SAXS curve has a few scattering peaks characteristic of cylindrical or spherical microdomains, while the SAXS curve of the solution-cast M62 has a single peak at a lower angle without any higher-order peaks, indicating that there is a large difference in the morphology between the solution-cast and quenched M62. That is, M62, which always has a crystallized microdomain structure (i.e., the microdomain structure in which PCL blocks crystallize partially) by quenching from the melt into any $T_{c}$, has a completely different morphology by the 


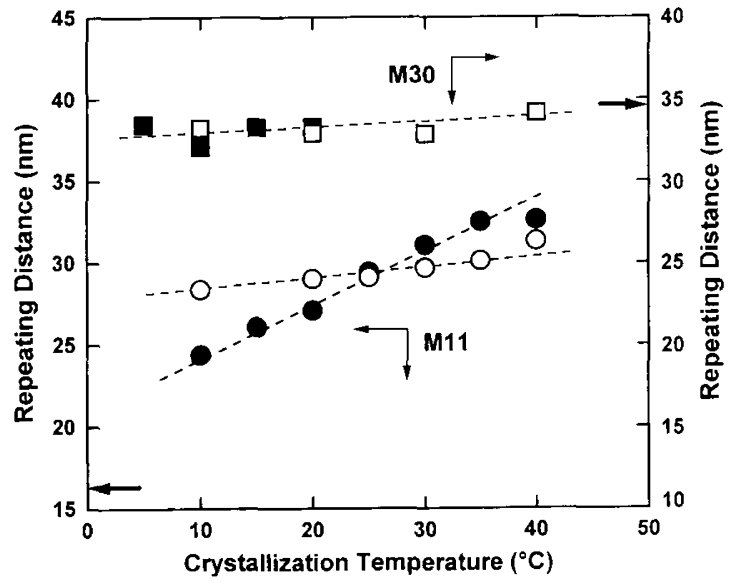

Figure 2. Repeating distance of the morphology formed in M11 $(\bigcirc, 0)$ and $M 30(\square, \square)$, evaluated from the angular position of the SAXS intensity peak, plotted against $T_{c}$. Open symbols represent the results of solution-cast samples and closed symbols quenched samples. The arrows on the ordinate represent the repeating distance of the microdomain structure in the melt.

solution-casting method at $T_{\mathrm{c}}=-20^{\circ} \mathrm{C}$. We can suppose it is the lamellar morphology because the crystallization of PCL blocks takes place slowly during the solvent evaporation to yield an equilibrium morphology (i.e., lamellar morphology) favorable for crystalline-amorphous diblock copolymers. ${ }^{2,3}$ The SAXS curve of the solutioncast M62 is also strongly dependent on $T_{\mathrm{c}}$; when $T_{\mathrm{c}}$ is higher than $10^{\circ} \mathrm{C}$ it is identical with that for the quenched M62 (the bottom curve in Figure 1). This fact indicates that the lamellar morphology appears at $T_{\mathrm{c}} \leq 0^{\circ} \mathrm{C}$ while the microdomain structure is maintained at $T_{\mathrm{c}} \geq 10^{\circ} \mathrm{C}$. The morphology formed in the solution-cast M44 and M140 is qualitatively similar to that of M62.

Figure 2 shows the $T_{\mathrm{c}}$ dependence of repeating distance $L$ in the lamellar morphology for the quenched (closed symbols) and solution-cast (open symbols) M11 and M30. $L$ for the quenched M11 increases significantly with increasing $T_{\mathrm{c}}$ (with the slope of $0.32 \mathrm{~nm}^{\circ} \mathrm{C}^{-1}$ ), as usually observed in the crystallization of homopolymers, which is ascribed to nonequilibrium crystallization at $T_{\mathrm{c}}$ in our previous paper. ${ }^{18} L$ for the quenched M30 is, on the other hand, almost independent of $T_{\mathrm{c}}$, probably because of the epitaxial relation between lamellar morphology and microdomain structure working during the crystallization and melting processes of crystallineamorphous block copolymers. ${ }^{25,26}$ Therefore the significant $T_{\mathrm{c}}$ dependence of $L$ cannot be observed in the quenched M30 as well as the solution-cast M30 because the final morphology will be mainly controlled by the epitaxial nature rather than the crystallization kinetics at $T_{\mathrm{c}}$.

We notice from Figure 2 that $L$ for the solution-cast M11 and M30 increases slightly with increasing $T_{\mathrm{c}}$. $(L$ for the solution-cast M44 and M62 also increases slightly, as shown in Figure 3.) The small increase of $L$ is reminiscent of the thermal expansion of microdomain structures reported by Sakamoto and Hashimoto for polystyrene-block-polyisoprene $\left(c a .0 .026 \mathrm{~nm}^{\circ} \mathrm{C}^{-1}\right)^{27}$ and by Register et al. for polyethylene-block-poly(3-methyl-1butene) (ca. $\left.0.041 \mathrm{~nm}^{\circ} \mathrm{C}^{-1}\right) .^{6}$ The present results for the

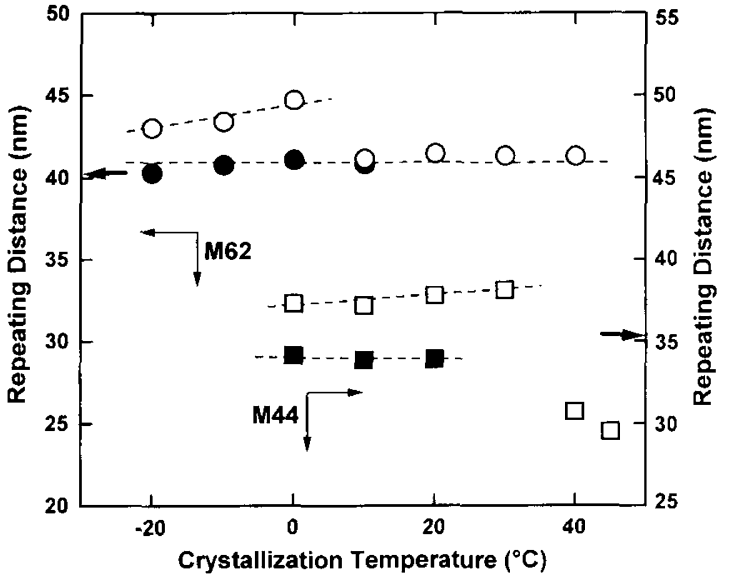

Figure 3. Repeating distance of the morphology formed in M44 $(\square, \square)$ and $\mathrm{M} 62(\bigcirc, \bigcirc)$ plotted against $T_{\mathrm{c}}$. Open symbols represent the results of solution-cast samples and closed symbols quenched samples. The arrows on the ordinate represent the repeating distance of the microdomain structure in the melt.

lamellar morphology are $0.061 \mathrm{~nm}^{\circ} \mathrm{C}^{-1}$ for $\mathrm{M} 11$ and $0.037 \mathrm{~nm}^{\circ} \mathrm{C}^{-1}$ for $\mathrm{M} 30$, which are comparable to the thermal expansion of the microdomain structure and much smaller than the value observed for the lamellar morphology formed under nonequilibrium crystallization $\left(=0.32 \mathrm{~nm}^{\circ} \mathrm{C}^{-1}\right)$, suggesting that the slight increase in $L$ for the solution-cast M11 and M30 does not originate from the morphology formation under nonequilibrium condition at $T_{\mathrm{c}}$ but from the thermal expansion of the resulting lamellar morphology (mainly composed of amorphous PB blocks). This fact indicates that $L$ for the solution-casting method is the equilibrium value at each $T_{\text {c }}$, and $L$ for the quenched M11 does not correspond to the equilibrium morphology, that is, a nonequilibrium effect intervenes in the quenching method during crystallization at $T_{\mathrm{c}}$ to yield the appreciable variation of $L$ from the equilibrium value.

Figure 3 shows the $T_{\mathrm{c}}$ dependence of $L$ for the quenched (closed symbols) and solution-cast (open symbols) M44 and M62, where $L$ at lower $T_{\mathrm{c}}\left(T_{\mathrm{c}} \leq 30^{\circ} \mathrm{C}\right.$ for $\mathrm{M} 44$ and $T_{\mathrm{c}} \leq 0^{\circ} \mathrm{C}$ for M62) is significantly larger than the repeating distance of microdomain structures $D$ (indicated by arrows and also by closed symbols) while it is smaller than $D$ for M44 and close to $D$ for M62 at higher $T_{\mathrm{c}}$. From the quantitative analysis of SAXS curves it is found that the microdomain structure is completely retained at higher $T_{\mathrm{c}}$ and PCL blocks crystallize partially within it while it is transformed into another morphology at lower $T_{\mathrm{c}}$, probably lamellar morphology as described previously. The temperature range where the morphological transition (microdomain structure $\rightarrow$ lamellar morphology) occurs by the crystallization contracts with increasing $M_{n}$; this transition occurred at all $T_{\mathrm{c}}$ for M11 and M30 while it was observed only at $T_{\mathrm{c}} \leq 30$ ${ }^{\circ} \mathrm{C}$ for $\mathrm{M} 44, T_{\mathrm{c}} \leq 0{ }^{\circ} \mathrm{C}$ for $\mathrm{M} 62$, and $T_{\mathrm{c}} \leq-10{ }^{\circ} \mathrm{C}$ for $\mathrm{M} 140$. These experimental facts reflect a complicated morphology formation during the solvent evaporation, and we will consider the mechanism of this morphology formation on the basis of phase diagram for this system to explain the present SAXS and DSC results.

Figure 4 shows the full width at half maximum 


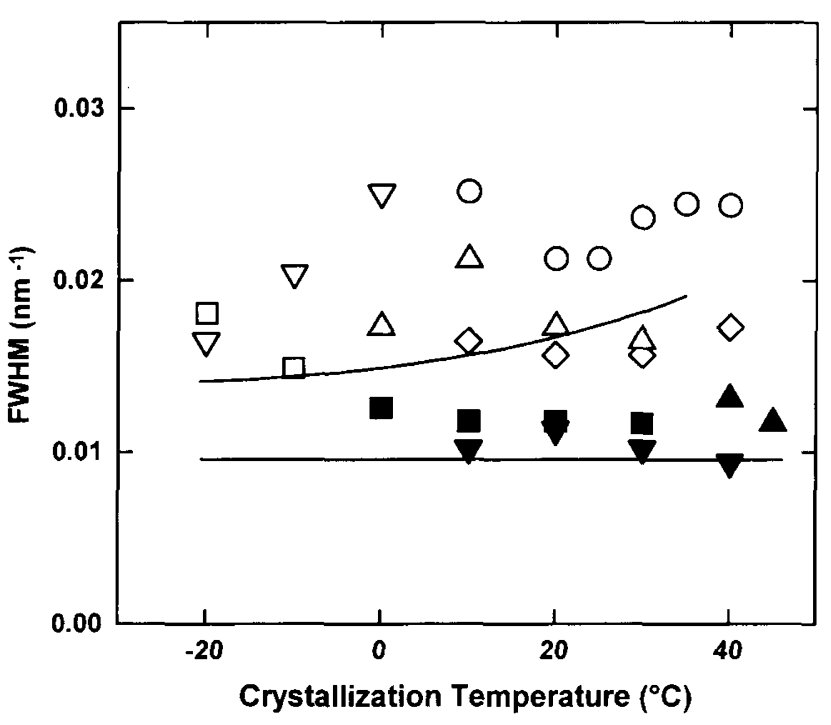

Figure 4. FWHM of the SAXS intensity peak for the solution cast M11 $(\bigcirc), \mathrm{M} 30(\diamond), \mathrm{M} 44(\triangle, \Delta), \mathrm{M} 62(\nabla, \boldsymbol{\nabla})$, and M140( $\square, \boldsymbol{\square}$ plotted against $T_{\mathrm{c}}$. Closed symbols represent that the PCL block crystallizes within the microdomain structure. Solid curves show the results of the quenched samples.

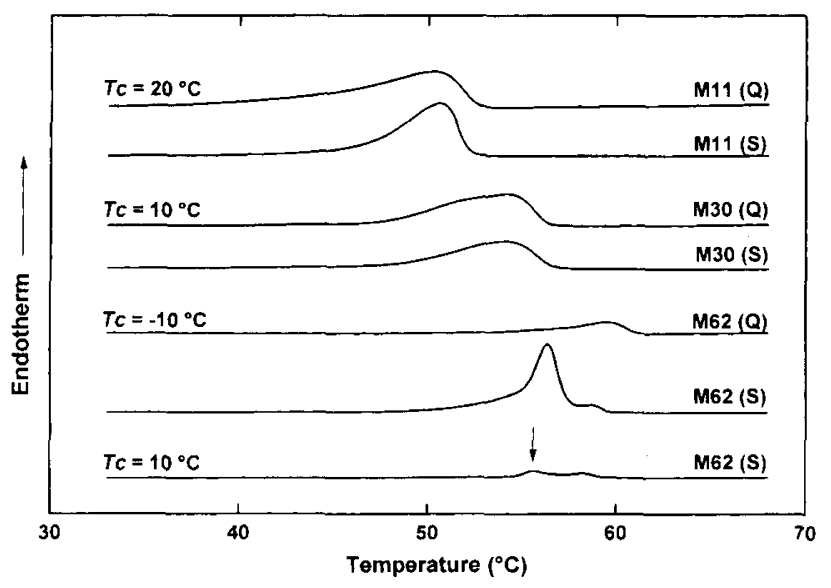

Figure 5. DSC endothermic curves of M11, M30, and M62 cast from the toluene solution (denoted by $\mathrm{S}$ ) and quenched from the microphase separated melt (Q). The heating rate is $5^{\circ} \mathrm{C} \min ^{-1} . T_{c}$ represents the crystallization temperature. The arrow indicates the major endothermic peak from which $T_{\mathrm{m}}$ was evaluated.

(FWHM) of the principal SAXS intensity peak plotted against $T_{\mathrm{c}}$, where FWHM is a measure of regularity for the morphology formed in the system; FWHM becomes smaller with increasing the regularity. The lamellar morphology formed by the solution-casting method has larger FWHM values than those for the crystallized microdomain structure (closed symbols) and also for the lamellar morphology and crystallized microdomain structure formed by quenching (solid curves) though the data points are considerably scattered. We cannot find any significant differences among the samples. The large FWHM value for the solution-cast samples suggests that the lamellar morphology is more irregular than that formed by the quenching method, which is counterintuitive for us because the solution-casting method makes

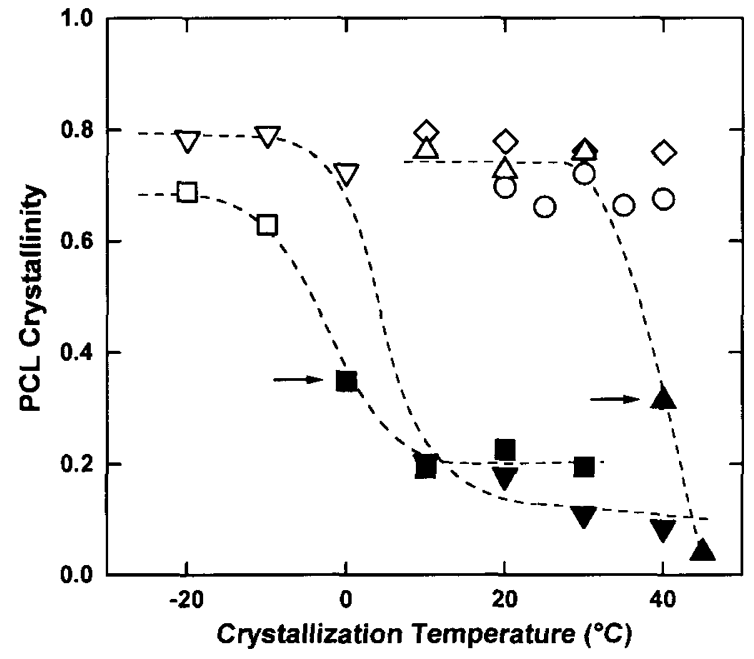

Figure 6. PCL crystallinity (the weight fraction of crystallized PCL blocks against total PCL blocks existing in the system) plotted against $T_{c}$ for $\mathrm{M} 11(\bigcirc), \mathrm{M} 30(\diamond), \mathrm{M} 44(\triangle, \mathbf{\Delta}), \mathrm{M} 62(\nabla, \boldsymbol{\nabla})$, and M140( $\square, \square)$. Closed symbols represent that the PCL block crystallizes within the microdomain structure. The data indicated by the arrow are obtained from the bimodal DSC curve suggesting the coexistence of microdomain structure and lamellar morphology.

the crystallization process slower to lead more regular morphology (or equilibrium morphology). It is necessary to clarity this point by further studies.

\section{DSC Measurements}

The typical DSC thermograms of M11, M30, and M62 are shown in Figure 5, where the results of the solutioncast and quenched samples are compared with each other. It is remarkable that two DSC curves for M62 crystallized at $T_{\mathrm{c}}=-10^{\circ} \mathrm{C}$ are extremely different; the DSC endothermic peak for the quenched M62 (which has the crystallized microdomain structure) is small while that for the solution-cast M62 (which has the lamellar morphology) is large comparable to those of M11 and M30. This means that $\chi_{c}$ is extremely different between two morphologies (lamellar morphology and crystallized microdomain structure), suggesting the substantial difficulty of crystallization within the curved microdomain. In addition, the DSC curve for the solution-cast M62 (and also M44 and M140) changes dramatically with changing $T_{\mathrm{c}}$; when $T_{\mathrm{c}}$ is high $\left(T_{\mathrm{c}} \geq 10^{\circ} \mathrm{C}\right)$ the endothermic peak is small (the bottom curve in Figure 5) while $T_{\mathrm{c}}$ is low $\left(T_{\mathrm{c}} \leq 0^{\circ} \mathrm{C}\right)$ it is large comparable to the DSC curves for PCL- $b$-PB with low $M_{n}$.

In Figure $6, \chi_{\mathrm{c}}$ is plotted against $T_{\mathrm{c}}$ for all the samples investigated, where open symbols represent that the lamellar morphology is formed by the crystallization of PCL blocks. The microdomain structure may coexist with a small amount of lamellar morphology in M44 at $T_{\mathrm{c}}=40^{\circ} \mathrm{C}$ and $\mathrm{M} 140$ at $T_{\mathrm{c}}=0^{\circ} \mathrm{C}$ (indicated by arrows) because the DSC curve shows a bimodal endothermic peak. We can find from Figure 6 that $\chi_{\mathrm{c}}$ decreases dramatically with increasing $T_{\mathrm{c}}$ for M44, M62, and M140 while it is constant at around 0.75 for M11 and M30. The $T_{\mathrm{c}}$ dependence of $\chi_{\mathrm{c}}$ shows that the transition temperature between low and high $\chi_{\mathrm{c}}$ values increases with decreasing $M_{n}$, where the morphology formed in the system 
(crystallized microdomain structure or lamellar morphology) is responsible for the magnitude of $\chi_{\mathrm{c}}$. That is, M62 has the lamellar morphology favorable for crystalline block copolymers at $T_{\mathrm{c}} \leq 0^{\circ} \mathrm{C}$ (corresponding to high $\chi_{\mathrm{c}}$ ) while it has the crystallized microdomain structure at $T_{\mathrm{c}} \geq 10^{\circ} \mathrm{C}$ (corresponding to low $\chi_{\mathrm{c}}$ ). The complicated morphology formation will be explained as a function of $M_{n}$ and $T_{\mathrm{c}}$ in the next section on the basis of phase diagram for this system.

Figure 7 shows the melting temperature $T_{\mathrm{m}}$ of PCL blocks, where closed symbols again represent the crystallized microdomain structure and open symbols the lamellar morphology. The value of $T_{\mathrm{m}}$ is almost constant $\left(55 \pm 3^{\circ} \mathrm{C}\right)$ irrespective of $T_{c}$ except that of M11 though $T_{\mathrm{m}}$ of crystalline homopolymers increases steadily with increasing $T_{\mathrm{c}}$. It is surprising that we cannot find any significant difference in $T_{\mathrm{m}}$ between two morphologies formed by the solution-casting method, in particular, those for M62 and M140. It is general to consider that PCL crystals within the microdomain structure will be immature and therefore the lamellar thickness is thin to give the low $T_{\mathrm{m}}$. But the experimental result shows the similar $T_{\mathrm{m}}$ both for the PCL blocks in the microdomain structure and lamellar morphology, suggesting the similar degree of lamellar thickness between two morphologies. The size of spherical or cylindrical microdomains may affect the crystallization because the microdomain with finite curvature, which depends intimately on the size, is considered to be disadvantageous to the crystallization of PCL blocks within it. We are now trying to elucidate the conformation of PCL blocks within the crystallized microdomain as a function of the spherical size. $^{28}$

\section{DISCUSSION}

To understand the mechanism of morphology formation during the solvent evaporation, it is useful to consider the phase diagram of a ternary system consisting of PCL, PB, and toluene with the crystallization and microphase separation regions, as shown in Figure 8, where the line connecting $\mathrm{PCL}$ and $\mathrm{PB}$ represents pure PCL- $b$-PB copolymers with various PCL fractions. This phase diagram is depicted at a fixed $T_{\mathrm{c}}$ and the PCL- $b$ $\mathrm{PB}$ solution proceeds from point $\mathrm{A}$ (initial composition of the PCL- $b$-PB solution) to point F (solution-cast PCL- $b$ $\mathrm{PB}$ with no toluene) during the solvent evaporation.

The limit of microphase separation is shown by the dotted curve in Figure 8, which is expected to shift upward (or toward the dilute PCL- $b$-PB solution) with increasing $M_{n}$. This is because the order-disorder transition temperature of microphase separation moves to the higher temperature, or lower polymer concentration in the case of polymer solutions, with increasing $M_{n}$, as predicted by recent theories for amorphous-amorphous diblock copolymers, ${ }^{29}$ and also verified by many experimental results. ${ }^{30}$ Therefore, the $M_{n}$ dependence of the limit of microphase separation arises from the thermodynamic factor working on block copolymers. The crystallization of PCL blocks occurs during the solvent evaporation and the limit of this crystallization shifts downward (or toward the concentrated PCL- $b$-PB solution) with increasing $T_{\mathrm{c}}$. This is because the crystalliza-

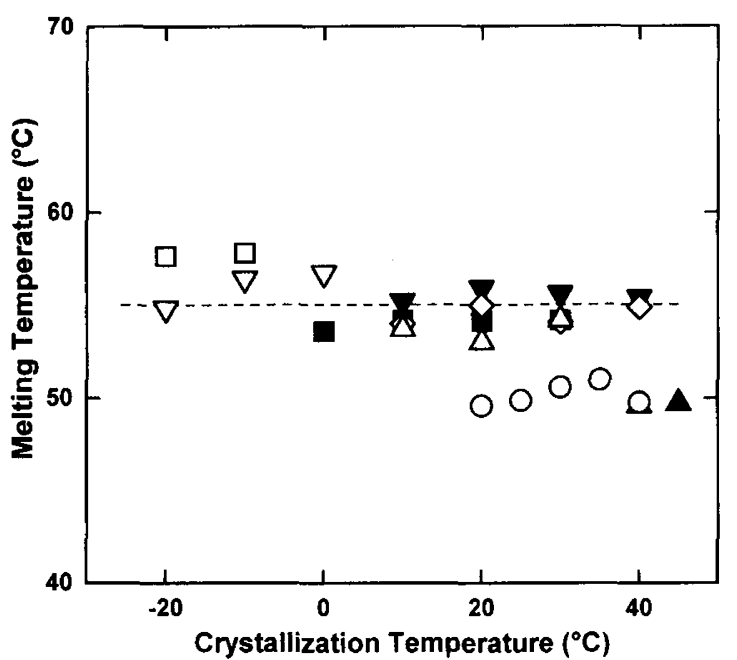

Figure 7. Melting temperatures plotted against $T_{c}$ for $\mathrm{M} 11(\mathrm{O})$, M30( $\diamond), M 44(\triangle, \Delta), M 62(\nabla, \nabla)$, and M140( $\square, \square)$. Closed symbols represent that the PCL block crystallizes within the microdomain structure. The horizontal dotted line is the average of all the data except those of M11.

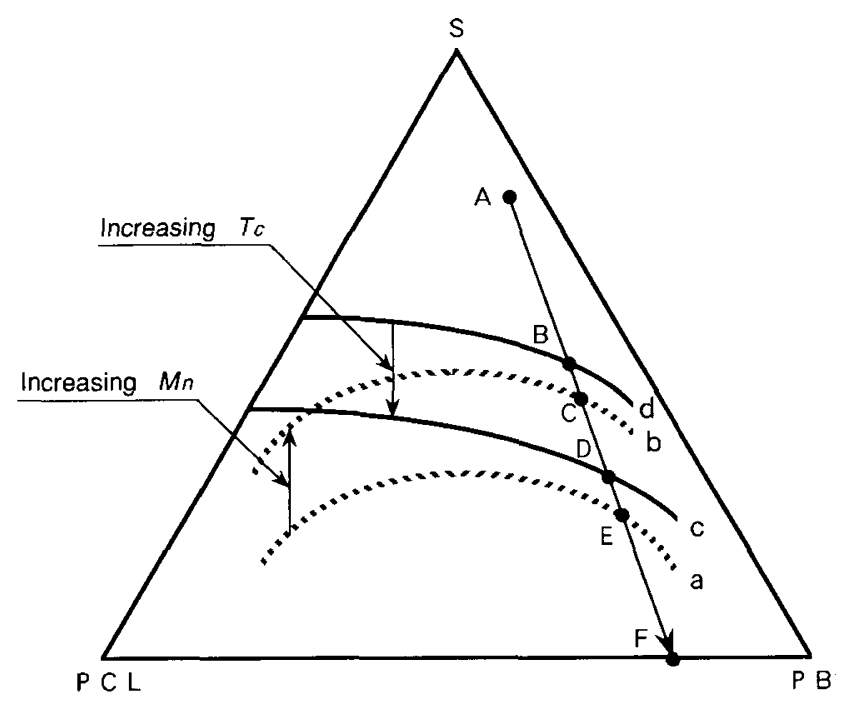

Figure 8. Ternary phase diagram composed of PCL, PB, and toluene schematically showing the relation between crystallization and microphase separation regions. The limit of microphase separation (broken curve) shifts upward with increasing $M_{n}$ and the limit of crystallization (solid curve) shifts downward with increasing $T_{c}$. Therefore the relative position of the microphase separation and crystallization regions changes with changing $M_{n}$ and $T_{\mathrm{c}}$.

tion rate of PCL blocks decreases and simultaneously the solvent evaporation rate increases with increasing $T_{\mathrm{c}}$. That is, the $T_{\mathrm{c}}$ dependence of the limit of crystallization is mainly ascribed to the kinetic factor arising from a competitive effect between solvent evaporation and crystallization. The relative positions of the microphase separation and crystallization regions on the phase diagram are shown in Figure 8 when $M_{n}$ and $T_{\mathrm{c}}$ change independently.

First we consider the case of PCL- $b$-PB copolymers with lower $M_{n}$, where the limit of microphase separation (curve a) is located at the higher PCL- $b$-PB concentration, so that during the solvent evaporation $(\mathrm{A} \rightarrow \mathrm{F})$ the 
PCL- $b$-PB solution meets the limit of crystallization (curve $\mathbf{c}$ or $\mathbf{d}$ ) before reaching curve $\mathbf{a}$ irrespective of $T_{\mathrm{c}}$. This means that the crystallization of PCL blocks occurs prior to microphase separation and eventually the lamellar morphology, favorable for crystalline-amorphous diblock copolymers, prevails in the system to yield high $\chi_{\mathrm{c}}$. This is the case for M11 and M30, and the SAXS and DSC results of M11 and M30 are explained by this mechanism of morphology formation during the solvent evaporation.

With increasing $M_{n}$ of PCL- $b$-PB, the limit of microphase separation shifts to the lower PCL- $b$-PB concentration (curve $\mathbf{a} \rightarrow$ curve $\mathbf{b}$ ) by the thermodynamic factor mentioned above. When $T_{\mathrm{c}}$ is high (curve c) the PCL- $b$ $\mathrm{PB}$ solution meets the limit of microphase separation (curve b) first during the solvent evaporation to yield microdomain structure in the system. The PCL block may crystallize partially within the microdomain structure (crystallized microdomain structure) to give low $\chi_{\mathrm{c}}$. When $T_{\mathrm{c}}$ is low (curve d), on the other hand, the PCL- $b$ PB solution meets the limit of crystallization (curve d) before reaching the limit of microphase separation (curve b). As a result, the lamellar morphology is formed again in the system, as in the case of PCL- $b$-PB copolymers with lower $M_{n}$, and therefore $\chi_{c}$ is high. This is the case for M44, M62, and M140, and we can successfully explain the complicated $T_{\mathrm{c}}$ dependence of $L$ (Figure 3 ) and $\chi_{\mathrm{c}}$ (Figure 6 ) for high molecular weight PCL- $b$-PB copolymers on the basis of the ternary phase diagram in which the limit of crystallization depends intimately on $T_{\text {c. }}$

The morphology formed in the solution-cast PCL- $b$-PB can be understood qualitatively by the complicated interplay between crystallization and microphase separation during the solvent evaporation as a function of $M_{n}$ and $T_{\mathrm{c}}$. Therefore, the morphological control of crystalline-amorphous block copolymers seems to be possible by changing $M_{n}$ and/or $T_{\mathrm{c}}$ by considering the phase diagram of the system, where the relative position of crystallization and microphase separation regions is important to control the final morphology formed in the system.

\section{CONCLUSIONS}

The morphology formed in poly ( $\varepsilon$-caprolactone)-blockpolybutadiene (PCL- $b$-PB) copolymers by casting the toluene solution has been investigated by SAXS and DSC as a function of total molecular weight $M_{n}(11000 \leq$ $\left.M_{n} \leq 140000\right)$ and crystallization temperature $T_{\mathrm{c}}(-20 \leq$ $T_{\mathrm{c}} \leq 45^{\circ} \mathrm{C}$ ). The morphology evaluated was quantitatively compared with that formed by quenching from the microphase-separated melt. The mechanism of morphology formation during the solvent evaporation was discussed in terms of the competition between crystallization and microphase separation on the basis of phase diagram for this system. The following conclusions were obtained.

1 . When $M_{n}$ was less than 30000 , the lamellar morphology was obtained at any $T_{\mathrm{c}}$ by the solution-casting method as well as the quenching method. The repeating distance of the resultant lamellar morphology was little affected by $T_{\mathrm{c}}$ for the solution-cast PCL- $b$-PB while it in- creased significantly with increasing $T_{\mathrm{c}}$ for the quenched PCL- $b$-PB, suggesting that the lamellar morphology formed in the solution-cast PCL- $b$-PB is the equilibrium morphology, while that formed in the quenched PCL- $b$ $\mathrm{PB}$ is the nonequilibrium morphology mainly determined by the crystallization kinetics at $T_{\mathrm{c}}$ though it may be close to the equilibrium one.

2. When $M_{n}$ was larger than 44000 , the solution-cast PCL- $b$-PB had the lamellar morphology or crystallized microdomain structure depending on $T_{\mathrm{c}}$ while the crystallized microdomain structure was always observed in the quenched PCL- $b$-PB. The PCL crystallinity $\chi_{\mathrm{c}}$ was extremely different between two morphologies; $\chi_{c}$ was large $\left(0.63 \leq \chi_{\mathrm{c}} \leq 0.79\right)$ and comparable to that of PCL homopolymer for the lamellar morphology and small $\left(\chi_{\mathrm{c}}<0.23\right)$ for the crystallized microdomain structure, suggesting the substantially difficulty of crystallization in the curved microdomain. The crystallization temperature at which the lamellar morphology formed shifted to the lower range with increasing $M_{n}$. The consideration of morphology formation revealed that the final morphology was determined by a complicated interplay between two factors; microphase separation thermodynamically affected by $M_{n}$ and crystallization mainly controlled by the kinetic factor at $T_{c}$.

Acknowledgment. This work was supported in part by Tokuyama Science Foundation and by Grants-in-Aid for Scientific Research (No. 12650879) from the Ministry of Education, Science, Sports, and Culture of Japan. A. $R$. acknowledges the financial assistance from Heiwa Nakajima Foundation and Association of International Education.

\section{REFERENCES}

1. I. W. Hamley, "The Physics of Block Copolymers", Oxford University Press, Oxford, 1998.

2. E. A. DiMarzio, C. M. Guttman, and J. D. Hoffman, Macromolecules, 13, 1194 (1980).

3. M. D. Whitmore and J. Noolandi, Macromolecules, 21, 1482 (1988).

4. S. Nojima, K. Kato, S. Yamamoto, and T. Ashida, Macromolecules, 25, 2237 (1992).

5. S. Nojima, S. Yamamoto, and T. Ashida, Polym. J., 27, 673 (1995).

6. D. J. Quiram, R. A. Register, and G. R. Marchand, Macromolecules, 30, 4551 (1997).

7. I. W. Hamley, J. P. A. Fairclough, F. S. Bates, and A. J. Ryan, Polymer, 39, 1429 (1998).

8. E. Hirata, T. Ijitsu, T. Soen, T. Hashimoto, and H. Kawai, Polymer, 16, 249 (1975).

9. M. Gervais and B. Gallot, Makromol. Chem., 178, 2071 (1977).

10. J. J. Herman, R. Jerome, P. Teyssie, M. Gervais, and B. Gallot, Makromol. Chem., 182, 997 (1981).

11. J. Heuschen, R. Jerome, and P. Teyssie, J. Polym. Sci., B27, $523(1989)$

12. K. C. Douzinas, R. E. Cohen, and A. F. Halasa, Macromolecules, 24, 4457 (1991).

13. R. Unger, D. Beyer, and E. Donth, Polymer, 32, 3305 (1991).

14. K. C. Douzinas and R. E. Cohen, Macromolecules, 25, 5030 (1992).

15. A. J. Lovinger, B. J. Han, F. J. Padden, and P. A. Mirau, J. Polym. Sci., B31, 115 (1993).

16. S. Ishikawa, S. Sasaki, and T. Fukutomi, J. Appl. Polym. Sci., 48, 509 (1993).

17. L. Liu, B. Jiang, and E. Zhou, Polymer, 37, 3937 (1996). 
18. A. Rohadi, R. Endo, S. Tanimoto, S. Sasaki, and S. Nojima, Polym. J., 32, 602 (2000).

19. S. Nojima, D. Wang, and T. Ashida, Polym. J., 23, 1473(1991).

20. S. Nojima, H. Nakano, Y. Takahashi, and T. Ashida, Polymer, 35, 3479 (1994).

21. S. Nojima, K. Hashizume, A. Rohadi, and S. Sasaki, Polymer, 38, 2711 (1997).

22. S. Nojima, M. Kuroda, and S. Sasaki, Polym. J., 29, 642 (1997).

23. S. Nojima, H. Tanaka, A. Rohadi, and S. Sasaki, Polymer, 39, 1727 (1998).

24. V. Crescenzi, G. Manzini, G. Galzolari, and C. Borri, Eur.
Polym. J., 8, 449 (1972).

25. I. W. Hamley, J. P. A. Fairclough, N. J. Terrill, A. J. Ryan, P. M. Lipic, F. S. Bates, and E. T. Andrews, Macromolecules, 29, 8835 (1996).

26. A. J. Ryan, J. P. A. Fairclough, I. W. Hamley, S. M. Mai, and C. Booth, Macromolecules, 30, 1723 (1997).

27. N. Sakamoto and T. Hashimoto, Macromolecules, 28, 6825 (1995).

28. S. Nojima, S. Hara, S. Tanimoto, and S. Sasaki, manuscript in preparation.

29. L. Leibler, Macromolecules, 13, 1602 (1980).

30. S. Sakurai, Trends in Polym. Sci., 3, 90 (1995). 